werden zuletzt in gepolsterte Weißblechdosen eingelegt, und die Dosen zum Schutz gegen Lichteinwirkungen verschlossen (Abb. 2). Zur weiteren Sicherheit werden die verschlossenen Dosen in einer Tiefkühltruhe bei $-30^{\circ} \mathrm{C}$ aufbewahrt. Diese Dosen können aber auch ohne weiteres tagelang bei Zimmertemperatur gelagert oder auch befördert werden. Die Haltbarkeit der auf diese Weise konservierten Hautstücke ist für die Dauer von etwa 3 Jahren gesichert. Zur weiteren Verbesserung der Lagerfähigkeit ist noch daran gedacht, in die Dosen ein sauerstoffverzehrendes Mittel (Glucoseoxydase) einzuführen, um das Eindringen von Sauerstoff in die Nähe des Aufbewahrungsglases zu verhindern. Das Glas kann auch in einer Vakuumdose aufbewahrt werden.

Bei der Verwendung konservierter Hautstücke für Transplantationen wird die getrocknete Haut für etwa 2 Std in physiologische Kochsalzlösung oder destilliertes Wasser gelegt, damit sie ihre frühere Form wieder erhält. Dann können die Hautstücke ohne weiteres überpflanzt werden. Dieses geschilderte Verfahren ist für die Konservierung der Haut durchaus notwendig. Wir haben derartig konservierte Hautstücke bei Verbrennungen wie üblich verpflanzt. Das Ergebnis der Verpflanzung entspricht natürlich den bisherigen Erfahrungen mit homoioplastischen Hautübertragungen. Wir sind aber bestrebt, durch besondere Methoden bei der Konservierung der Haut die üblichen Abwehrreaktionen nach der Verpflanzung auszuschalten. Über die Ergebnisse dieser Forschungen hoffen wir, bald berichten zu können.

Vorsitzender: Ich danke Herrn Budrass. Ich fand es richtig, daß wir uns einmal einen Einblick in unsere kleine „Konservenfabrik" im ,Bergmannsheil" in Bochum haben geben lassen. In Amerika arbeiten Riesenstäbe mit Millionen von Dollars. Wir haben den ím Verhältnis dazu geringen Betrag von etwa $40000 \mathrm{DM}$ zur Verfügung, mit dem wir versuchen, weiterzukommen. Vielleicht gelingt es.

Ich darf nun Frau WinkLeR und Herrn Mildesie bitten. Es spricht Frau WINKLER.

\title{
5. Zur Frage der Fremdhautreaktion
}

\section{Von}

\author{
F. Winkler und H. Millest-Wien \\ (Vortragende: Frau E. WINKLER)
}

Mit den grundlegenden Arbeiten von Medevar wurden eine Reihe von Tierexperimenten veröffentlicht, die darauf hinweisen, daß bei der Abstoßung von Fremdhauttransplantaten immunologische Vorgänge ursächlich zumindest mitbeteiligt sind. Auch beim Menschen sprechen verschiedene Anhaltspunkte für diese Annahme. Andererseits fehlt es nicht an gegenteiligen Ansichten. So stoßen die verwendeten Versuchs- 